\title{
CONTRIBUTO AL CALCOLO DELL'ASSORBIMENTO NELLA PROPAGAZIONE IONOSFERICA DELLE ONDE CORTE
}

\author{
A. Bolle - P. Dominici
}

I. - Le questioni concernenti l'assorbimento delle radioonde nella propayazione ionosferica occupano un posto di grande importanza nel quarlro generale delle ricerche ionosferiche. I dati ricavati dalle misure dell'assorbimento ionosferico integrano infatti in maniera pressoche completa i dati forniti dai normali sondaggi. Essi danno un contributo particolarmente notevole allo studio dello strato $D$ ed allo studio della dipendenza esistente fra i fenomeni ionosferici ed i fenomeni greomagnetici e solari. Per questa ragione, ed anche per la noterole importanza pratica della questione per ciò che riguarda le radiocomunicazioni a grande distanza, si è dato ovunque grande impulso alla esecuzione di esperienze e misure sull'assorbimento ionosferico.

In questa memoria svolgeremo alcune considerazioni teoriche indispensabili all'analisi ed alla corretta interpretazione delle misure di assorhimento da noi eseguite e che verranno a suo tempo illustrate. Piiu precisamente calcoleremo il coefficiente di attenuazione ionosferica per le onde corte, e quindi l'assorbimento competente ai vari -trati ionosferici ed alle varie modalita di propagazione dell'onda.

Tabella dei simboli piü frequentemente usati.

i unità immayninaria

c velocità della luce nel vuoto $=3.10^{10} \mathrm{~cm} / \mathrm{sec}$

(1) pulsazione dell'onda

$f$ frequenza dell'onda

$n$ indice di rifrazione della ionosfera

$\varepsilon$ costante dielettrica della ionosfera

$\sigma$ conducibilità elettrica della ionosfera

$\gamma \quad$ coefficiente di attenuazione ionosferica

I' assorbimento ionosferico

$m$ massa dell'elettrone $=9,04 \cdot 10^{\circ 8}$ gr 
e carica elettrica dell'elettrone $=4,77 \cdot 10^{-10}$ ues CGS

$V$ densità elettronica $=$ elettroni $\mathrm{cm}^{3}$

$v \quad$ frequenza delle collisioni fra elettroni liberi e molecole neutre $=$ collisioni $/ \mathrm{sec}$

${ }^{(1)}$ pulsazione giromagnetica $=\frac{e H}{m c}$

$H$ intensità del campo geomagnetico

i) angolo fra la direzione del campo geomagnetico e la direzione di propagazione dell'onda

$\left.\omega_{\mathrm{L}}=(1)_{\mathrm{II}} \cdot \cos \mathrm{i}\right)$

$\left.(1)_{\mathrm{T}}={ }^{(1)}\right)_{\mathrm{II}} \cdot \operatorname{sen} i \mathrm{~T}$

$L=\frac{k T}{m g}$ è la scala delle altezze

$\omega_{0}^{2}=\frac{4 \pi N e^{2}}{m}$

Definizione del coefficiente di attenuazione ionosferica.

2. - Consideriamo un'onda piana sinusoidale di pulsazione (:) clie si propaghi nella ionosfera. Se assumiamo l'asse $x$ del triedro di riferimento $(0, x, y, z)$ nella direzione di propagazione dell'onda in modo che il vettore campo elettrico sia diretto lungo l'asse $z$, l'equlizione dell'onda si può scrivere:

$$
E_{\mathrm{z}}=E_{\mathrm{o}} e^{j(1)\left(t-\frac{q}{c} x\right)}
$$

L'indice di rifrazione $q$ che compare in questa espressione $\grave{e}$ in genere un numero complesso, suscettibile di essere messo sotto questa forma:

$$
q=n-j k
$$

Con questa posizione la [1] si trasforma nella:

$$
E_{\mathrm{z}}=E_{\mathrm{o}} e^{-k \frac{11}{c} x} \cdot e^{j^{(1)\left(\ell-\frac{n}{c} x\right)}}
$$


Questa formula mostra che l'onda si propaga con una velocità di fase $v=\frac{c}{n}$ e quindi $n$ è l'indice di rifrazione della ionosfera; l'anpiezza:

$$
E=E_{\mathrm{o}} e^{-\gamma}
$$

decresce esponenzialmente con l'aumentare della distanza percorsa, secondo il coefficiente:

$$
\gamma=\frac{(1) k}{c}
$$

che pertanto chiameremo coefficiente di attenuazione ionosferica.

Se indichiamo con $S$ l'intero tragitto dell'onda, l'intensità di camfo all'arrivo sarà passata dal valore $E_{0}$ al valore:

$$
E=E_{0} e^{-\Gamma}
$$

arendo indicato con $\Gamma$ l'assorbimento subito, pari a:

$$
\Gamma=\int_{s} \gamma d s
$$

dove $d s$ i l'elemento di traieltoria generico di $S$.

Vediamo ora di esplicitare $\gamma$ dalla [5].

3. - In un primo momento trascuriamo l'influenza del campo geomagnetico sulla propagazione dell'onda.

In base alle equazioni di Maxwell possiamo scrivere le seguenti relazioni :

$$
\left\{\begin{array}{l}
\frac{\partial H_{y}}{\partial x}=4 \pi \sigma F_{z}+\varepsilon \frac{\partial F_{z}}{\partial t} \\
\frac{\partial \tilde{H} y}{\partial t}=c^{2} \frac{\partial F_{z}}{\partial x}
\end{array}\right.
$$

Derivando lia prima rapporto a $t$, la seconda rapporto ad $x$, abbiamo:

$$
\frac{\partial^{2} F_{z}}{\partial x^{2}}=4 \pi \sigma \frac{\partial F_{z}}{\partial t}+\varepsilon \frac{\partial^{2} E_{z}}{\partial t^{2}}
$$

Calcolandoci le rerivate della [1] e sostituendole nella precedente:

$$
q=\varepsilon-j \stackrel{4 \pi \sigma}{(1)}
$$


e, ricordando la [2]:

$$
(n-j k)^{2}=\varepsilon-j \frac{4 \pi \pi}{(1)}
$$

da cui :

$$
\left\{\begin{array}{l}
n^{0}-k^{2}=\varepsilon \\
n k=\frac{2 \pi \sigma}{\omega}
\end{array}\right.
$$

sistema che, risolto, ci dà la soluzione:

$$
\begin{aligned}
& n=\sqrt{\frac{\varepsilon}{2}+\sqrt{\frac{\varepsilon^{2}}{4}+\frac{4 \pi^{2} \tau^{2}}{\omega^{2}}}} \\
& k=\sqrt{-\frac{\varepsilon}{2}+\sqrt{\frac{\varepsilon^{2}}{4}+\frac{4 \pi^{2} \sigma^{2}}{\omega^{2}}}}
\end{aligned}
$$

Eे:

$$
\sqrt{\frac{\varepsilon^{2}}{4}+\frac{4 \pi^{2} \sigma^{2}}{\omega^{2}}}=\frac{\varepsilon}{2} \sqrt{1+\frac{16 \pi^{2} \sigma^{2}}{\varepsilon^{2}()^{2}}}
$$

Con uno sviluppo in serie arrestato al primo termine:

Si ha così :

$$
k=\frac{2 \pi \sigma}{\omega \sqrt{\varepsilon}}
$$

$$
\gamma=\frac{2 \pi \sigma}{c\rceil_{\varepsilon}}
$$

Operando analogamente sulla [8]:

$$
n=\sqrt{\varepsilon}
$$

ed in definitiva:

$$
\gamma=\frac{2 \pi \sigma}{c n}
$$

A questo punto occorre precisare come varino nella ionosfera le due qualità $n$ e $\sigma$ in funzione delle quali $\gamma$ è espresso.

4. - La presenza di particelle elettrizzate nell'alıa atmosera ioni ed elettroni - modifica profondamente le caratteristiche elettriche della stessa per due motivi:

1) intanto, il movimento di vibrazione forzata impresso a dette 
particelle dal campo elettrico dell'onda incidente provoca, come vedremo, una variazione del valore della costante dielettrica;

2) in secondo luogo, a causa degli urti fra le particelle elettrizzate e le circostanti molecole neutre si ha una dissipazione di energia a spese dell'onda incidente.

In questi fenomeni le particelle che hanno una parte preponderante sono gli elettroni piuttosto che gli ioni, a causa della molto minore massa dei primi.

Si abbia dunque una regione ionosferica con densità elettronica $N$, sottoposta all'azione di un campo alternato:

$$
E_{\mathrm{z}}=E_{\mathrm{o}} \cos \omega t
$$

Ogni eletirone è sottoposto ad una accelerazione:

$$
\frac{d^{2} z}{d t^{\circ}}=\frac{e}{m} E_{0} \cos \omega t
$$

ed acquista una velocità :

$$
\frac{d z}{d t}=\frac{e}{m \omega} E_{\mathrm{o}} \operatorname{sen}(1) t
$$

Ogni elettrone dà così luogo ad una corrente:

$$
e \cdot \frac{d z}{d t}=-\frac{e^{2}}{m \omega^{2}} \cdot \frac{d E}{d t}
$$

complessivamente si ha una densità di corrente in quadratura con la tensione:

$$
-\frac{N e^{2}}{m \omega^{2}} \cdot \frac{d E}{d t}
$$

Se non ci fossero stati elettroni liberi, la densità di corrente di spostamento sarebbe stata:

Ora invece si ha:

$$
\frac{d D}{d t}=\frac{1}{4 \pi} \cdot \frac{d E}{d t}
$$

$$
\frac{d L^{\prime}}{d t}=\left(\frac{1}{4 \pi}-\frac{N e^{2}}{m \omega^{2}}\right) \cdot \frac{d E}{d t}
$$

e quindi la costante dielettrica è passata dal valore 1 al valore:

$$
\varepsilon=1-\frac{4 \pi N \epsilon^{2}}{m \omega^{2}}
$$


e l'indice di rifrazione al valore:

$$
n=\sqrt{1-\frac{4 \pi N e^{2}}{m \omega^{2}}}
$$

5. - In realtà le cose sono complicate dagli urti fra elettroni liberi e molecole neutre. Infatti ciò che interviene a modificare la costante dielettrica $\grave{e}$, in sostanza, che gli elettroni acquistano una velocità in quadratura col campo elettrico dell'onda. Ora, a causa degli urti, varia in modo del tutto casuale la fase della velocita acquistata dagli elettroni rispetto al campo. Si tratta allora di calcolare il valor medio della componente in quadratura col campo della velocità degli $N$ elettroni contenuti nell'unità di volume quando la fase della velocità rispetto al campo elettrico dellonda varia comunque fra 0 e $2 \pi$.

Nell'istante $t=o$ l'elettrone subisca un urto, nell'istante $t_{0}$ il successivo; sia $v_{z}$ la componente lungo l'asse $z$ della velocitì acquistata dall'elettrone in dipendenza dell'urto.

Se :

$$
E_{\mathrm{z}}=E_{\mathrm{o}} \cos (\omega t+\varphi)
$$

è il campo elettrico dell'onda, la velocità dell'elettrone in $11 n$ istante qualsiasi fra due urti successivi è:

$$
\frac{\partial z}{d t}=\frac{e E_{0}}{m \omega}\left[\operatorname{sen}(\omega t+(1)-\operatorname{sen} \varphi]+v_{z}\right.
$$

Possiamo sviluppare questa funzione in serie trigonometrica di Fourier, e ricavarci il termine che ci interessa, quello cioe in quadratura col campo, la cui ampiezza $V\left(t_{0}, \mathfrak{\varphi}\right)$ è:

$$
\left.r\left(t_{0}, \varphi\right)=\frac{2}{t_{0}} \int_{0}^{t_{0}}\left\{\frac{e F_{0}}{m(1)}[\operatorname{sen}(\omega t+\gamma)-\operatorname{sen} \varphi]+v_{z}\right\} \operatorname{sen}(\omega) t+\varphi\right) d t
$$

cioè :

$$
\begin{gathered}
V\left(t_{\mathrm{o}}, \varphi\right)=\frac{2}{t_{\mathrm{o}}}\left\{-\frac{v_{\mathrm{z}}}{\omega}\left[\cos \left(\omega t_{\mathrm{o}}+\varphi\right)-\cos \varphi\right]+\frac{e E_{-}}{m \omega}\right. \\
\left.\left[\frac{t_{\mathrm{o}}}{2}-\frac{\operatorname{sen} 2\left((1) t_{\mathrm{n}}+\varphi\right)}{4(1)}-\frac{\operatorname{sen} 2(\varphi}{4(1)}+\frac{\operatorname{sen} \varphi \cos \left(\omega t_{\mathrm{o}}+\varphi\right)}{\omega}\right]\right\}
\end{gathered}
$$


Il valore medio rispetto alla fase è:

$$
V\left(t_{0}\right)=\frac{1}{2 \pi} \int_{0}^{2 \pi} V\left(t_{0}, \varphi\right) d \varphi
$$

Danno contributo nullo a $V\left(t_{0}\right)$ tutti i termini dell'espressione precedente contenenti sen $\varphi, \cos \varphi$, sen $\varphi \cos \uparrow$; rimangono solo i due termini in $t_{0}$ e $\operatorname{sen}^{2} p$ sen $\omega t_{0}$. Si ba:

$$
V\left(t_{\mathrm{o}}\right)=\frac{e F_{\mathrm{o}}}{m \omega}\left(1-\frac{\operatorname{sen} \omega t_{\mathrm{c}}}{\omega t_{\mathrm{o}}}\right)
$$

Se $u_{z}$ è la componente lungo l'asse $z$ della velocità media degli elettroni per agitazione termica, al tempo $t_{0}$ corrisponde un cammino libero $z=u_{\mathrm{z}} t_{\mathrm{o}}$. Sappiamo dalla teoria cinetica dei gas che, chiamando con $l$ il cammino medio libero, la probabilità che un elettrone abbia un percorso libero compreso fra $z \mathrm{e} z+d z \dot{\mathrm{e}}$ :

$$
e^{-\frac{z}{l}} \frac{d z}{l}
$$

Allora la probabilità di azione per la componente $V\left(t_{0}\right)$ è:

$$
\frac{z}{l} \cdot \frac{1}{l} e^{--\frac{z}{l}} d z
$$

ed il valore medio di $V\left(t_{0}\right)$ rispetto a tutti i possibili tempi di liberó percorso $\dot{\mathrm{e}}$ :

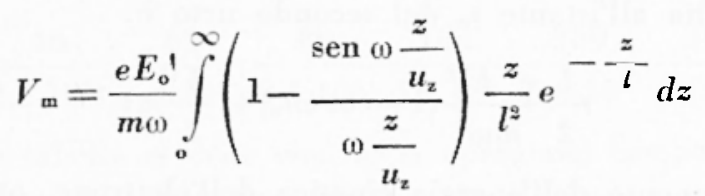

Ricordando i due integrali notevoli:

$$
\int_{0}^{\infty} x e^{--x} d x=1 ; \int_{0}^{\infty} \operatorname{sen} q x e^{-p x} d x-\frac{q}{p^{2}+q^{2}}\left(^{*}\right)\left(^{* *}\right)
$$

(*) Cauchy, Cours de lę̧ons. $\quad\left(^{* *}\right) x=\frac{z}{u_{z}}, q=\omega, p=\frac{u_{\mathrm{z}}}{i}$ 
l'integrazione indicata è immediata, e fornisce:

$$
V_{\mathrm{m}}=\frac{e E_{\mathrm{o}}}{m} \cdot \frac{\omega}{\omega^{2}+v^{2}}
$$

avendo posto $\frac{u_{\mathrm{z}}}{l}=v$, frequenza degli urti.

Rifacendo i calcoli più sopra svolti, per una velocità degli elettroni pari a $V_{\mathrm{m}}$, si trova per l'indice di rifrazione il valore:

$$
n=\sqrt{1-\frac{4 \pi N e^{2}}{m\left(\omega^{2}+v^{2}\right)}}
$$

6. - Vediamo ora l'entità dell'energia sottratta al campo elettrico dell'onda e dissipata negli urti.

Intanto si ha, in un istante qualunque fra due urti successivi:

$\left\langle\frac{d z\rangle^{2}}{d t} !^{2}=\frac{e^{2} E_{\mathrm{o}}{ }^{2}}{m^{2} \omega^{2}}\{\operatorname{sen}(\omega t+\varphi)-\operatorname{sen} \varphi]^{2}+2 \frac{e E_{\mathrm{o}}}{m \omega} v_{\mathrm{z}}[\operatorname{sen}(\omega t+\varphi)-\operatorname{sen} \varphi]+v_{\mathrm{z}}{ }^{2}\right.$

Cerchiamone il valor medio. Intanto, il valor medio di $v_{z}^{2}$ è $\bar{v}_{s}^{2}$ velocità quadratica media, ed il valor medio di $v_{z}$ è zero. Quanto al primo termine si ha:

$$
[\operatorname{sen}(\omega t+\varphi)-\operatorname{sen} \varphi]^{2}=\operatorname{sen}^{2}(\omega t+\varphi)-2 \operatorname{sen} \varphi \operatorname{sen}(\omega t+\varphi)+\operatorname{sen}^{2} \varphi
$$

La media di ognuno dei due termini quadrati per $\varphi$ che varia comunque fra 0 e $2 \pi$ è $1 / \underline{\text { }}$. Il doppio prodotto si scrive:

$-2 \operatorname{sen} \varphi \operatorname{sen}(\omega t+\varphi)=-2 \operatorname{sen} \varphi \cos \varphi \operatorname{sen}(\omega t+\varphi)-2 \operatorname{sen}^{2} \varphi \cos (\omega t+\varphi)$

Il valore medio del primo termine è zero; complessivamente l'energia cinetica media all'istante $t_{0}$ del secondo urto è:

$$
\frac{1}{2} \frac{e^{2} E_{\mathrm{o}}^{2}}{m \omega^{2}}\left(1-\cos \omega t_{\mathrm{o}}\right)+\frac{1}{2} m \bar{v}^{2}
$$

Dunque l'aumento dell'energia cinetica dell'elettrone, ottenuto a syese del campo elettrico dell'onda è:

$$
W=\frac{1}{2} \frac{e^{2} F_{\mathrm{o}}^{2}}{m \omega^{2}}\left(1-\cos \omega t_{\mathrm{o}}\right)
$$

Al solito, al tempo $t_{0}$ corrisponde un libero percorso $z=u_{z} t_{0}$, ed il valor medio dell'energia dissipata negli urti dagli $N$ elettroni dell'unità di volume si ottiene considerando tutti i possibili liberi percorsi : 


$$
W_{\mathrm{m}}=\frac{1}{2} \frac{N^{2} r_{\mathrm{o}}}{m \omega^{2}} \int_{0}^{\infty}\left(1-\cos \omega \frac{z}{u_{\mathrm{z}}}\right) \frac{1}{l} e^{-\frac{z}{l}}, z
$$

L'integrazione si effettua mediante i due integrali notevoli:

$$
\int_{0}^{\infty} e^{-x} d x=1 ; \int_{0}^{\infty} \cos q x e^{-p x} d x=\frac{p}{p^{2}+q^{2}}
$$

e si ha:

$$
H^{\prime}=\frac{1}{2} \frac{N e^{2} E_{0}}{m} \cdot \frac{1}{(1)^{2}+v^{2}}
$$

Avendosi $v$ urti al secondo, l'energia dissipata in un secondo per gli urti è :

$$
\frac{1}{2} \frac{N^{2} E_{0}^{2}}{m} \cdot \frac{v}{(1)^{2}+v^{2}}
$$

Poiché la conducibilitá elettrica di un mezzo è il fattore o per cui occorre moltiplicare il quadrato del valore efficace del campo elettrico se si vuole avere l'energia dissipata nell'unita di tempo e di volume, si ha:

$$
\sigma\left(\frac{F_{\alpha}}{\left(\gamma^{2}\right)}\right)^{2}=\frac{1}{2} \frac{{ }^{\prime} e^{2} F_{\mathrm{s}}{ }^{2}}{m} \cdot \frac{v}{\omega^{2}+v^{2}}
$$

e quindi la conducibilita elettrica della ionosfera vale:

$$
\sigma=\frac{N \rho^{*}}{m} \cdot \frac{v}{\omega^{2}+v^{2}}
$$

Riportiamo qui di seguito una tabella che dá i valori di $v$ secondo Chapman per diverse altezze dal suolo.(2).

$\frac{\mathrm{Km}}{\mathrm{v}} \mid$\begin{tabular}{cccccccc}
20 & 40 & 60 & 80 & 100 & 200 & 300 & 500 \\
\hline $8,5.10^{10}$ & $2,5.10^{9}$ & $1,4.10^{8}$ & $8,5.10^{5}$ & $4,3.10^{5}$ & $8,5.10^{3}$ & $8,5.10^{2}$ & 2,8
\end{tabular}

Da questa tabella si vede che, se si opera nel campo delle onde corte, con onde ciò̀ di frequenza fra 3 e $30 \mathrm{Mhz}$, non si commette un grande errore trascurando nelle [12] e [13] $v^{2}$ di fronte a $\omega^{2}$ appena clie si considerino regioni ionosferiche di quota non inferiore ai 100 $\mathbf{k m}$ circa. In tale ipotesi per $n$ e $\sigma$ valgono le:

$$
n=\sqrt{1-\frac{4 \pi N e^{2}}{m \omega^{2}}}
$$

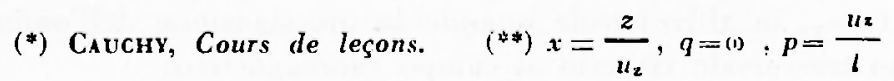




$$
\sigma=\frac{\Lambda_{1}^{2},}{m \omega^{2}}
$$

Concludiamo cosi che, se si trascura l'azione del campo geomagnetico sulla ionosfera, il coefficiente di attenuazione vale:

$$
\gamma=\frac{2 \pi N_{t}^{2}}{m c n} \cdot \frac{v}{\left(\omega^{2}+v^{2}\right.}
$$

ovvero, se $\omega^{2}>>v^{5}:$

$$
\gamma=\frac{2 \pi N e^{2}}{m c n} \cdot \frac{\because}{\omega^{2}}
$$

¡. - Vediamo ora di renderci conto dei limiti di validità della [15] testé stabilita quando si prenda in considerazione anche il campo geomagnetico.

I calcoli che precisano l'influenza del campo geomagnetico sulla propagazione di una radioonda nella ionosfera esulano dal tema di questo lavoro, e ci limitiamo perciò a darne i risultati. Precisamente, Appleton ( ${ }^{3}$ ) ha ricavato per l'indice di rifrazione complesso $q$ il valore:

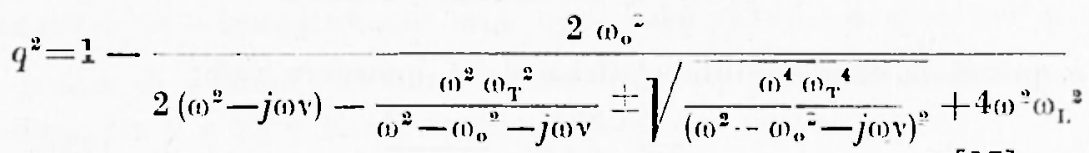

Il doppio segno del radicale che compare nella [17] significa che si hanno due diversi indici complessi di rifrazione, cioè l'onda, dal punto di vista più generale, si divide in due componenti.

I coefficienti di attenuazione relativi ad ognuna delle due componenti si ottengono separando nella [17] dalla parte reale il coefficiente dell'immaginario $k$, posto:

$$
q=n-j k
$$

Seguendo questa via si è però condotti ad espressioni estremamente complesse. E molto più conveniente eseguire le operazioni di calcolo ponendosi in una delle due seguenti condizioni:

1) quando nella [17] è lecito trascurare $i$ termini in ${ }^{(1)}{ }_{L}$ rispetto ai termini in $\omega_{\mathrm{T}}$, in altre parole quando la propagazione dell'onda si può ripetere trasversale rispetto al campo geomagnetico; 
2) quando viceversa è lecito trascurare $i$ termini in $\omega_{T}$ rispetto ai termini in (1) ${ }_{1}$ (propagazione quasi longitudinale).

In termini della [17] si è nella prima o nella seconda condizione se $\dot{e}$ rispettivamente $\frac{\omega^{4} \omega_{r^{4}}^{4}}{\left(\omega^{2}-\left(1_{0}{ }^{2}-j \omega v\right)^{2}\right.}$ maggiore o minore di $4 \omega^{*}{ }^{2} \omega_{L}{ }^{2}$, clie equivale alle:

$$
\begin{array}{rr}
\frac{\omega_{\varphi^{2}}^{2}}{2 \omega_{\mathrm{L}} v}>1 & \text { propagazione quasi trasversale } \\
\frac{\omega_{\mathrm{v}}{ }^{2}}{2 \omega_{\mathrm{I}} v}<1 & \text { propagazione quasi longitudinale }
\end{array}
$$

Nel caso di onde a tragito verticale, la $H_{\mathrm{L}}$ si riduce alla componente verticale del campo geomagnetico, la $H_{\mathrm{T}}$ alla componente orizzontale.

Nella tabella che segue diamo i valori del rapporto critico $\frac{(1)_{T}{ }^{2}}{2()_{L} v}$ calcolati a diverse quote per Pavia, Roma e Palermo, assumendo per $H$ i valori misurati nel giugno 1951.

$\begin{array}{lcccccc}\text { Km } & 40 & 60 & 30 & 100 & 150 & 200 \\ \text { Pavia } & 3,7.10^{-1} & 7,3.10^{-3} & 1,2.10^{-1} & 2,4 & 60 & 120 \\ \text { Roma } & 4,8.10^{-1} & 9,6.10^{-3} & 1,6.10^{-1} & 3,1 & 79 & 158 \\ \text { Palermo } & 6.10^{-1} & 1,21.0^{-:} & 1,91.0^{-1} & 3,9 & 98 & 196\end{array}$

Come si vede, alle nostre latitudini un'onda a tragitto verticale si propaga "quasi longitudinalmente " sino a circa $100 \mathrm{~km}$ dal suolo, "quasi trasversalmente " ad altezze superiori.

8. - a) Propagazione quasi trasversale.

La [17] si riduce alla:

$$
q^{0}=1-\frac{2 \omega_{0}^{2}}{2\left((1)^{2}-j(1) v\right)-\frac{\omega^{2} \omega_{T}^{2}}{(1)^{2}-\omega_{0}^{2}-j \omega v} \pm \frac{\omega^{2} \omega_{\mathrm{T}}^{2}}{\omega^{2}-\omega_{0}^{2}-j \omega v}}
$$

Prendendo il + del doppio segno:

Ricordando la [2]:

$$
q^{2}=1-\frac{\omega_{0}^{2}}{\omega^{2}-j \omega v}
$$

$$
(n-j k)^{2}=1-\frac{\left(\omega_{0}^{2}\right.}{(1)} \cdot \frac{(1)^{2}+j v}{\omega^{2}+v^{2}}
$$


Uguagliando le parti reali ed i coefficienti dell'immaginario:

$$
\begin{aligned}
& n^{2}-k^{2}=1-\frac{\omega_{0}^{2}}{\omega^{2}+v^{2}} \\
& n k=\frac{\omega_{0}^{2}}{2 \omega} \cdot \frac{v}{\omega^{2}+v^{2}}
\end{aligned}
$$

da cui si ricava :

$$
k=\frac{2 \pi N e^{2}}{m \cdot \omega n} \cdot \frac{v}{\omega^{2}+v^{2}}
$$

ed infine:

$$
\gamma=\frac{2 \pi N \epsilon^{2}}{m c n} \cdot \frac{v}{\omega^{2}+v^{2}}
$$

Il fatto di aver riottenuto la [15] significa che questa prima componente magnetoionica si propaga senza essere influenzata dal campo geomagnetico; si è convenuto di chiamarla raggio ordinario dell'onda, riservando il nome di raggio straordinario alla componente corrispondente al - del doppio segno.

Per quest'ultima si ha dalla [19]:

$$
q^{2}=1-\frac{\omega_{0}^{2}}{\omega^{2}-j(1) v-\frac{(1)^{2} \omega_{r}^{2}}{(1)^{2}-\omega_{0}^{2}-j \omega v}}
$$

che può ridursi alla seguente:

$$
q^{\prime \prime}=1-\frac{\omega_{0}^{2}}{(1)} \cdot \frac{(1)-j v}{((1)-j v)^{2}-(1)_{1}^{2}}
$$

Razionalizzando:

$$
q^{2}=1-\frac{\omega_{0} z}{\omega} \frac{\omega\left(\omega^{2}-\omega_{\mathrm{T}}{ }^{2}+v^{2}\right)+j v\left(\omega^{2}+\omega_{\mathrm{T}}{ }^{2}+v^{2}\right)}{\left(\omega^{2}-\omega_{\mathrm{T}}{ }^{2}-v^{2}\right)^{2}+4(1)^{2} v^{2}}=(n-j k)^{2}
$$

da cui :

$$
\left\{\begin{array}{l}
n^{2}-k^{2}=1-\omega_{0}^{2} \frac{(1)^{2}-\omega_{\mathrm{T}}+v^{2}}{\left(\omega^{2}-\omega_{\mathrm{T}^{2}}-v^{2}\right)^{2}+4 \omega^{2} v^{2}} \\
n k=\frac{\omega_{0}^{2} v}{\omega} \cdot \frac{\omega^{2}+\omega_{\mathrm{T}}^{2}+v^{2}}{\left((1)^{2}-\left(\omega_{\mathrm{T}}^{2}-v^{2}\right)^{2}+4\left(\omega^{2} v^{2}\right.\right.}
\end{array}\right.
$$


e quindi :

$$
\gamma=\frac{2 \pi N e^{\prime \prime}}{m c n} \cdot v \frac{\omega^{2}+\omega_{\mathbf{T}}^{2}+v^{2}}{\left(\omega^{2}-\omega_{T}^{2}-v^{2}\right)^{2}+4 \omega^{2} v^{2}}
$$

9. - b) Propagazione quasi longitudinale.

La [17] dà luogo alla:

$$
\underline{q}^{2}=1-\frac{\omega_{0}^{2}}{\omega^{2}+\omega \omega_{L}-j \omega v}
$$

Razionalizzando:

da cui :

$$
q^{2}=1-\frac{\omega_{0}{ }^{2}}{(1)} \cdot \frac{\omega \pm \omega_{L}+j v}{\left(\omega \pm \omega_{L}\right)^{2}+v^{2}}=(n-j \eta)^{2}
$$

e quindi :

$$
\left\{\begin{array}{l}
n^{2}-k^{2}=1-\frac{\omega_{0}^{2}}{\omega} \cdot \frac{\omega \pm \omega_{L}}{\left(\omega+\omega_{L}\right)^{2}+v^{2}} \\
n k=\frac{\omega_{0}^{2}}{2(1)} \cdot \frac{v}{\left(\omega \pm(1)_{L}\right)^{2}+v^{2}}
\end{array}\right.
$$

$$
\gamma=\frac{2 \pi N e^{2}}{m c n} \cdot \frac{\because}{\left.(\alpha)+\omega_{L}\right)^{\hat{*}}+v^{z}}
$$

le due componenti magnetoioniche corrispondendo una al + , una al -- del doppio segno.

Ora, se si opera con onde corte, $\omega_{\mathrm{L}}$ e $\omega_{\mathrm{T}}$ possono trascurarsi nei calcoli rispetto ad $\omega$, e si vede facilmente che le [20] e [21] si riducono ancora alla [15].

Calcolo dell'assorbimento ionosferico.

10. - Riprendendo la [7] proponiamoci di calcolare l'assorbimento subito da un'onda lungo un tragitto ionosferico $S$.

Col medesimo procedimento usato per il calcolo della rifrazione atmosferica, si può stabilire la seguente relazione:

$$
R n_{0} \operatorname{sen} i_{0}=(R+h) n \operatorname{sen} i
$$

dove $R$ è il raggio terrestre, $n_{0}$ e $i_{0}$ l'indice di rifrazione e l'angolo zenitale dell'onda al suolo, $n$ ed $i$ i corrispondenti valori alla quota $h$. 
Assumendo $n_{0}=1$ e dividendo per $R$ si ha:

$$
\operatorname{sen} i_{o}=\left(1+\frac{h}{R}\right) n \operatorname{sen} i
$$

E da notare che il rapporto $\frac{h}{h}$ non supera, per la normale propagazione ionosferica, il valore 0,08 ; cosicché la relazione precedente può gcriversi, in forma non rigorosa ma sufficientemente esatta:

$$
\operatorname{sen} i_{\mathrm{o}}=n \operatorname{sen} i
$$

D'altra parte la variazione di quota $d h$ è legata all'elemento generico di traiettoria $d s$ dalla relazione:

$$
d h=d s \cdot \cos i
$$

ovvero, ricavando $\cos i$ dalla [22]:

$$
d s=\frac{[n d h}{\sqrt{n^{2}-\operatorname{sen}^{2} i_{\mathrm{o}}}}
$$

Mediante le [23] e [15] si ha dalla [7]:

$$
\Gamma=\frac{2 \pi e^{2}}{m c}\left\lceil\frac{N v d h}{\left(\omega^{2}+v^{2}\right) \sqrt{n^{2}-\sec ^{2} r_{n}}}\right.
$$

Per eseguire l'integrazione indicata occorre esplicitare le due funzioni $N(h)$ e $v(h)$.

Secondo Chapman ( $\left.{ }^{4}\right)$ la funzione $N(h)$ ha la seguente forma:

$$
N=N_{0} e^{1 / 2\left[1-z-f(\varkappa) e^{*} \cdot\right]}
$$

dove :

$$
\begin{aligned}
& x \quad \text { è l'angolo zenitale del sole } \\
& f(x) \equiv \frac{1}{\cos x} \quad \text { per } \quad 0<x<75^{\circ} \\
& f(x) \equiv \sqrt{\frac{\pi}{2} \cdot \frac{h+R}{L}} \quad \text { per } \quad 75^{\circ}<x<90^{\circ}
\end{aligned}
$$

$N_{0}$ è la massima densità elettronica della regrione conside rata per $x=0$.

$z=\frac{h-h_{\mathrm{o}}}{L}$

$h_{0}$ è l'altezza alla quale $N$ assume il valore $N_{0}$. 
Quanto alla $v(h)$ si ha:

$$
v=v_{0} e^{-z}
$$

dove $v_{0} \grave{e}$ il valore che $v$ assume alla quota $h_{0}$.

Comparendo nella [25] l'indice di rifrazione $n$, conviene dividere il tragitto dell'onda nella ionosfera in tratti di due specie:

1) tratti percorsi senza notevoli deviazioni, cioè in regioni in cui $n$ si può assumere pari all'unità (regioni non deviatrici);

2) tratti in prossimità del punto di riflessione dell'onda (apice della traiettoria).

11. - In una regione non deviatrice, essendo $n=1$ si ha dalla [24], tenuto conto delle [25] e [26], per l'assorbimento subito dall'onda nella doppia traversata della regione:

$$
\Gamma_{1}=\frac{4 \pi e^{z}}{m c} \cdot \sec i_{0} \cdot e^{\frac{1}{2}} L N_{0} v_{0} \int^{+\infty o} \frac{e^{-\frac{1}{2}\left[z+f(x) e^{-z}\right]}}{\omega^{2}+v_{0}^{2} e^{-2 z}} d z
$$

Introducendo il rapporto $x=\frac{v}{\omega}$ fra la frequenza delle collisioni e la pulsazione dell'onda, la [27] si trasforma nella:

dove:

$$
\begin{aligned}
& \Gamma_{1}=\frac{4 \pi e^{2}}{m c} \cdot \sec i_{0} \cdot \frac{e^{\frac{l}{2}} L N_{0}}{\gamma \omega v_{0}} A \\
& A=\int_{0}^{\infty} \frac{x^{\frac{1}{2}}}{1+x^{2}} e^{-a x} d x \\
& \text { con: } \quad a=\frac{f(x)}{2} \cdot \frac{(1)}{v_{0}}
\end{aligned}
$$

Dai grafici che seguono si possono ricavare i valori di $A$ in funzione di (1), $v, f(x) . \quad(3)$.

Questa è la formula generale che permette di calcolare $\Gamma_{1}$ per una data regione ionosferica.

In particolare, per lo strato $E$ ed operando con onde corte è $\omega^{2}>>v^{2}$; se inoltre $v$ si assume costante al valore $v_{\mathrm{oE}}$, si ha dalla [27]: 


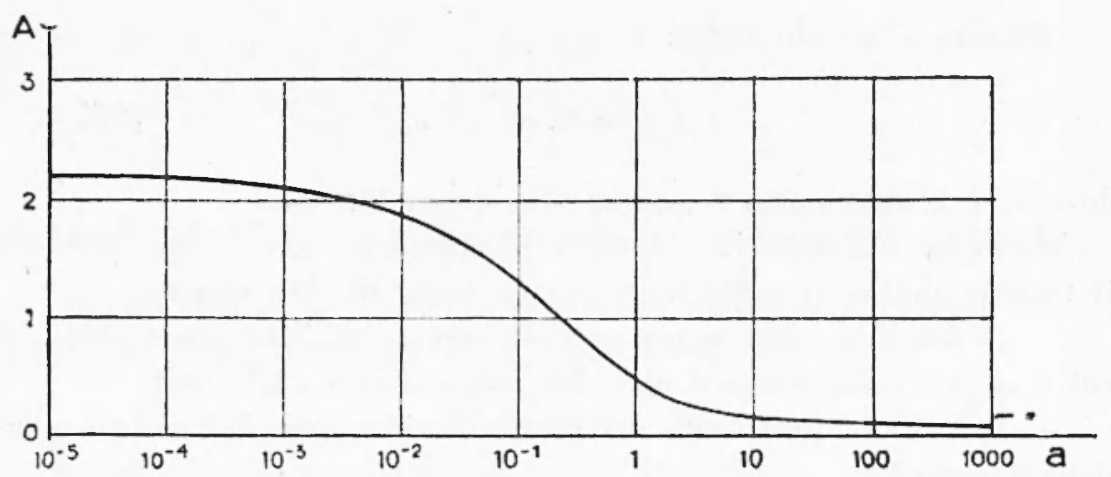

Fig. 1

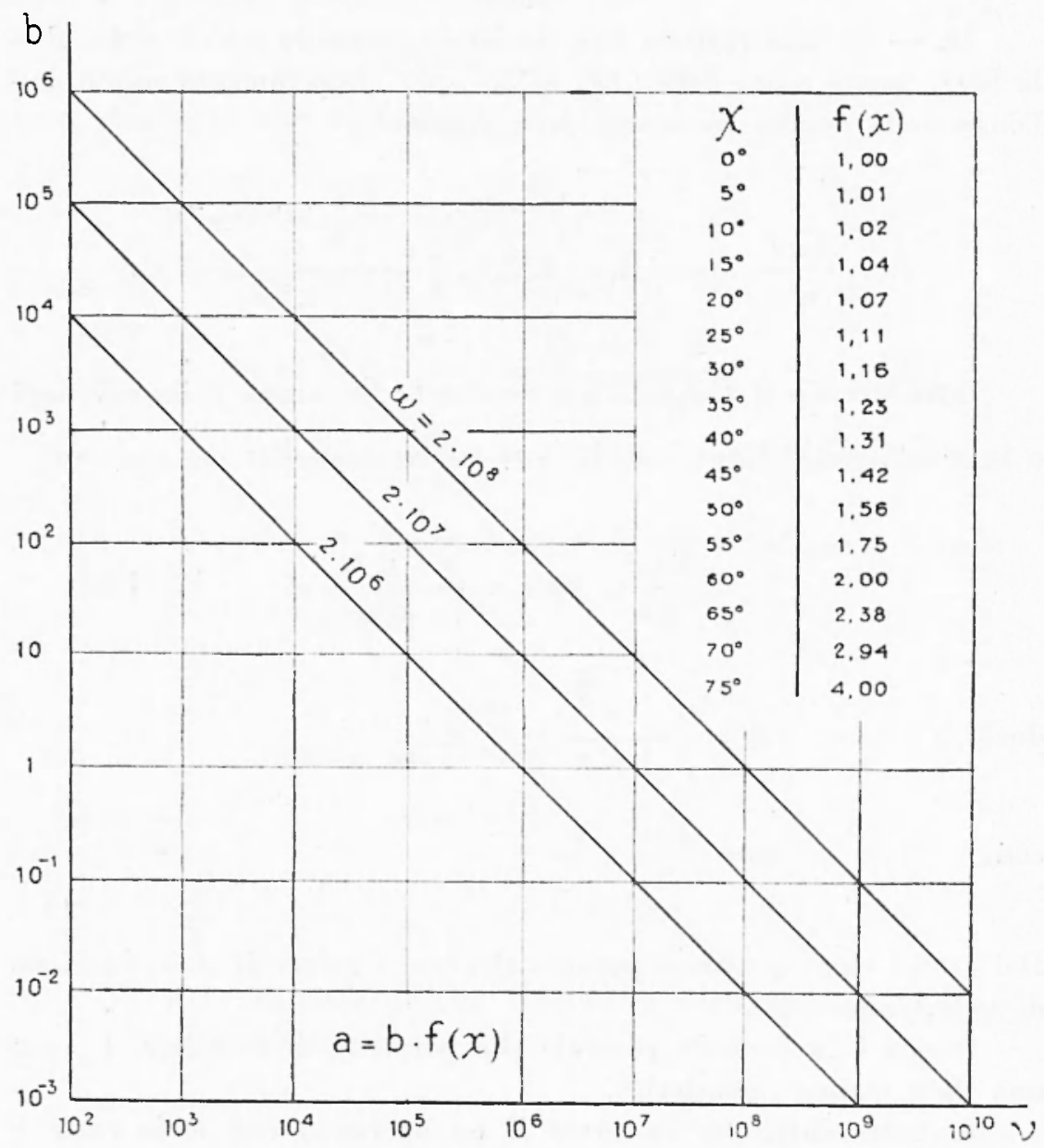

Fig- 2 


$$
I_{I E}=\frac{4 \pi e^{0}}{m c} \cdot \frac{\sec i_{0}}{\omega^{2}} \cdot e^{\frac{1}{2}} L N_{\mathrm{OE}} v_{\mathrm{OE}} \int_{-\mathrm{oO}}^{+\infty} e^{-\frac{1}{2}\left[z+f(x) e^{-z}\right]} d z
$$

Ponendo $x=\mathrm{e}^{-\underline{z}} \stackrel{\underline{z}}{-}$

$$
\begin{aligned}
& \int_{-\infty}^{+\infty o} e^{-\frac{1}{2}} d z=2 \int_{0}^{\infty} e^{-\frac{f(x)}{2} x^{2}} d x= \\
& =2 \sqrt{2}[f(x)]--\int_{2}^{1} \int_{0}^{\infty} e^{-y^{2}} d y=\sqrt{2 \pi}[f(x)]-\frac{1}{2}
\end{aligned}
$$

cioè :

$$
\Gamma_{1 \mathrm{~V}}=\frac{1 \pi \epsilon^{2}}{m c} \cdot \frac{\sec i_{\kappa}}{\omega^{*}} \cdot \gamma^{\prime} \overline{2 \pi e L} N_{\mathrm{OE}} v_{\mathrm{OE}}(\cos x)^{\frac{1}{2}}
$$

D'altra parte, la massima densità di ionizzazione $N_{\mathrm{OE}}$ si può esprimere in funzione della frequenza critica $f_{E}^{\prime}$ per incidenza normale:

$$
N_{\mathrm{OE}}=\frac{m \pi}{e^{2}}\left(f_{F}^{\prime}\right)^{2}
$$

Finalmente:

$$
\Gamma_{1 \mathrm{E}}=\frac{4,131}{c} v_{\mathrm{OE}}\left(f_{\mathrm{E}}^{\prime}\right)^{\bar{z}}(\cos x)^{-\frac{1}{2}} \cdot \frac{\sec i_{\mathrm{o}}}{J^{2}}
$$

Se invece non si assume costante al valore $v_{\mathrm{OE}}$ la frequenza delle collisioni nello strato, si vede facilmente che la [31] va modificata nella :

$$
\Gamma_{\mathrm{Il}}=\frac{4,13 L}{c} v_{\mathrm{OE}}\left(f_{\mathrm{E}}\right)^{2}(\cos \psi)^{\frac{3}{2}} \cdot \frac{\sec i_{\mathrm{o}}}{f^{2}}
$$

In ogni modo, l'assorbimento subito da un'onda che attraversa lo slrato $E$ viene ad essere inversamente proporzionale al quadrato della frecuenza dell'onda, ed aumenta con l'aumentare dell'angolo di incidenza dell'onda sullo strato. 
12. - In una regione deviatrice, vale a dire nella regione in cui ha luogo la riflessione dell'onda, si può generalmente ritenere che sia $\omega^{2}>>\gamma^{2}$, ove ci si limiti, come noi stiamo facendo, al campo delle onde corte. $\mathrm{La}[24]$ si riduce quindi alla:

$$
\Gamma_{0}=\frac{4 \pi e^{2}}{m c \omega^{2}} \int \frac{N v d h}{\sqrt{n^{2}-\operatorname{sen}^{2} i_{\mathrm{o}}}}
$$

Chiamiamo $N_{\mathrm{a}}$ la densità elettronica della regione all'apice della traiettoria dell'onda. E:

$$
N_{\mathrm{z}}=\frac{m(1)^{2}}{4 \pi e^{2}} \cos ^{2} i_{\mathrm{o}}
$$

Ricordando la [11] si ha:

$$
\sqrt{n^{\circ}-\operatorname{sen} i_{0}}=\sqrt{\cos ^{2} i_{0}-\frac{4 \pi e^{2}}{m(1)^{2}}}=\cos i_{0} \sqrt{1-\frac{N}{N_{2}}}
$$

Sostituendo nella [33]:

$$
\Gamma_{2}=\frac{4 \pi e^{2}}{m c \omega^{2}} \cdot \sec i_{0} \cdot \int_{:}^{h_{\mathrm{a}}} \frac{N v d h}{\sqrt{1-\frac{N}{N_{\mathrm{a}}}}}
$$

dove $h_{\mathrm{a}}$ è l'altezza dell'apice della traiettoria $\left({ }^{6}\right)$.

Assumiamo, per semplicità, $v$ costante in prossimità dell'apice. Per la funzione $N(h)$, alla [25] sostituiamo una più semplice disıribuzione di tipo parabolico:

$$
N=k h^{2}
$$

Osserviamo che è:

Si ha in conseguenza :

$$
h_{\mathrm{a}}=\sqrt{\frac{\overline{N_{\mathrm{a}}}}{k}}
$$

$$
\int_{0}^{h_{\mathrm{a}}} \frac{N d h}{\sqrt{1-\frac{N}{N_{\mathrm{a}}}}}=k h_{\mathrm{a}} \int_{0}^{h_{\mathrm{a}}} \frac{h^{2} d h}{\sqrt{h_{\mathrm{a}}{ }^{2}-h^{2}}}=\frac{\pi}{4} k h_{\mathrm{a}}{ }^{3}
$$


$\mathbf{E}:$

$$
\Gamma_{2}=\frac{\sqrt{\cdots \pi}}{8 e c} \cdot \frac{\omega v}{\sqrt{k}} \cdot \cos ^{2} i_{0}
$$

Esprimendo il coefficiente $k$ in funzione della frequenza critica $f^{\prime}$ dello strato e della corrispondente altezza di riflessione $h^{\prime}$ :

$$
\Gamma_{2}=\frac{\pi}{4 c} \cdot v \frac{h^{\prime}}{f^{\prime}} \cdot \cos i_{\mathrm{o}} \cdot f
$$

Questa formula mostra che, se è valida la [36], l'assorbimento subito dall'onda nella zona di riflessione è direttamente proporzionale alla frequenza dell'onda e diminuisce con l'aumentare dell'angolo di incidenza dell'onda sullo strato.

Con una distribuzione per $N$ di tipo esponenziale:

si ba :

$$
N=p e^{q h}
$$

$$
\int_{0}^{h_{\mathrm{a}}} \frac{N d h}{\sqrt{1-\frac{N}{N_{\mathrm{a}}}}}=p \int_{0}^{h_{\mathrm{a}}} \frac{e^{q h} d h}{\sqrt{1-e^{q\left(h-h_{\mathrm{a}}\right)}}}==\frac{N_{\mathrm{a}}}{q_{0}} \int_{0}^{1} \frac{d y}{\sqrt{y}}-\frac{N_{\mathrm{a}}}{q}
$$

Sostituendo e ricordando la [34]:

$$
\Gamma_{n}=\frac{2 v}{c q} \cdot \cos i_{\mathrm{o}}
$$

dipendente non più dalla frequenza dell'onda ma solo dall'inclinazione dell'onda sullo strato e dalle caratteristiche dello strato stesso, che intervengrono mediante $v$ e soprattutto mediante il coefficiente $q$ della (38).

Secondo Namba e Tsukada ( $(\overrightarrow{)})$, che hanno dato per l'assorbimento complessivo $\Gamma$ una formula empirica del tipo:

$$
\Gamma=\sqrt{\cos x}\left(\frac{a}{f^{2}}+b f\right)
$$

si dovreblue ritencre come più probabile l'ipotesi clıe ha condotto alla [37]. Naturalmente però, per pronunciarci con sicurezza su questo ed altri punti alquanto ipotetici dei nostri calcoli, occorrerà attendere $\mathrm{i}$ dati ricavati dalle misure.

Roma - Istituto Nazionale di Geofisica - Giugno 1952. 


\section{RIASSUNTO}

Come premessa alla illustrazione delle misure di assorbimento ionosferico eseguite, si svolgono le necessarie considerazioni teoriche. Viene calcolato il valore del coefficiente di attenuazione ionosferica per le onde corte ed il valore dell'assorbimento competente ai vari strati ionosferici ed alle varie modalità di propagazione dell'onda.

\section{SUMMARY}

Before presenting some measurements of ionospheric absorption, we would like to present some necessary theoretical considerations for the understanding of these measurements.

The value for the coefficient of atmospheric attenuation for short waves, the absorption applying to the various ionospheric layers and to the various modes of propagation of the waves have been calculated.

\section{BIBLIOGRAFIA}

(1) Jounust, L'ionosphère. Paris 1946.

(2) Chapman, Quart. Journal of Roval Met. Soc. Vol. 46 pag. 357.

(3) Appleton, Journ of Inst. of Elect. Eng. Vol. 71, pag. 642.

(4) Chapman, Proc. Phis. Soc. Vol. 43, pag. 26, pag. 483.

(5) Appleton, Proc. Roy. Soc. Vol. 162, pag. 451.

(6) Namba e Tsukada, Proc. Inst. Radio Eng. Vol, 2l, pag. 245.

(7) Namba e Tsukada, Proc. Inst. Radio Eng. Vol. 21, pag. 1013. 